\title{
The safety and efficacy of immunotherapy with anti-programmed cell death 1 monoclonal antibody for lung cancer complicated with Mycobacterium tuberculosis infection
}

\author{
Jinpeng Shi ${ }^{1,2,3 \#}$, Jiayu Li ${ }^{1 \#}$, Qi Wang ${ }^{1,2,3}$, Xiaomin Cheng ${ }^{1,2,3}, \mathrm{He} \mathrm{Du}^{1}$, Ruoshuang Han ${ }^{1,3}$, Xuefei $\mathrm{Li}^{4}$, \\ Chao Zhao ${ }^{4}$, Guanghui Gao ${ }^{1}$, Yayi He ${ }^{1}$, Xiaoxia Chen ${ }^{1}$, Chunxia Su ${ }^{1}$, Shengxiang Ren ${ }^{1}$, Fengying $\mathbf{W u}^{1}$, \\ Zhemin Zhang ${ }^{2}$, Caicun Zhou ${ }^{1}$
}

${ }^{1}$ Department of Medical Oncology, Shanghai Pulmonary Hospital \& Thoracic Cancer Institute, School of Medicine, Tongji University, Shanghai, China; ${ }^{2}$ Department of Pulmonary \& Critical Care Medicine, Shanghai Pulmonary Hospital, School of Medicine, Tongji University, Shanghai, China; ${ }^{3}$ School of Medicine, Tongji University, Shanghai, China; ${ }^{4}$ Department of Lung Cancer and Immunology, Shanghai Pulmonary Hospital, School of Medicine, Tongji University, Shanghai, China

Contributions: (I) Conception and design: F Wu, J Shi, J Li; (II) Administrative support: Z Zhang, C Zhou; (III) Provision of study materials or patients: G Gao, Y He, X Chen, C Su, S Ren; (IV) Collection and assembly of data: X Cheng, H Du, R Han; (V) Data analysis and interpretation: Q Wang, X Li, C Zhao; (VI) Manuscript writing: All authors; (VII) Final approval of manuscript: All authors.

\#These authors contributed equally to this work.

Correspondence to: Prof. Fengying Wu. Department of Medical Oncology, Shanghai Pulmonary Hospital \& Thoracic Cancer Institute, School of Medicine, Tongji University, No. 507, Zheng Min Road, Shanghai 200433, China. Email: fywu@163.com; Prof. Zhemin Zhang. Department of Pulmonary \& Critical Care Medicine, Shanghai Pulmonary Hospital, School of Medicine, Tongji University, No. 507, Zheng Min Road, Shanghai 200433, China. Email: zhemindoc@163.com.

Background: Anti-programmed cell death 1 (PD-1)/programmed cell death-ligand 1 (PD-L1)
immunotherapy has boosted the prognosis in advanced lung cancer. Meanwhile, accumulating cases showed
the correlation between tuberculosis (TB) reactivation and anti-PD-1/PD-L1 immunotherapy. However,
the safety and efficacy of anti-PD-1/PD-L1 immunotherapy for lung cancer complicated with TB infection
could only be learned from real-world data. Methods: We retrospectively analyzed 562 patients with advanced lung cancer who received anti-PD-1/ PD-L1 immunotherapy at Shanghai Pulmonary Hospital from 2015 to 2019, including 13 patients with TB infection. Besides, relevant literature reviews were performed online to analyze the safety and efficacy of immunotherapy and to explore the appropriate treatment strategies in this specific population.

Results: In our cohort, the initiation of anti-PD-1/PD-L1 immunotherapy was from June 2015 to December 2019. Among them, 13 patients had TB infection prior to immunotherapy including 11 latent TB and 2 active TB, and all of them were treated with anti-PD-1 immunotherapy. Patients with active TB infection were treated with concurrent anti-TB and anti-PD-1 treatments, and the remaining received either mono-immunotherapy or combined immunotherapy. Neither reactivation of latent TB nor progression of active TB was monitored in our cohort during immunotherapy. Severe immune-related adverse events (irAEs) were diagnosed in two patients. Treatment strategies such as discontinuation of immunotherapy and administration of corticosteroids were provided timely, and one with latent TB infection got gradually improved, but the other one with active TB died quickly. The median progression-free survival (PFS) was 5.5 months for tumor immunotherapy in our cohort. However, the PFS of immunotherapy was merely 2.1 and 2.2 months in lung cancer patients with active TB infection.

Conclusions: Immunotherapy is relatively safe for lung cancer patients complicated with previously treated latent $\mathrm{TB}$, and the efficacy of immunotherapy in this specified population is not inferior to that in lung

^ ORCID: 0000-0001-5140-3238. 
cancer patients without TB infection. TB screening before anti-PD-1/PD-L1 immunotherapy is strongly recommended, and irAEs should be monitored more cautiously in lung cancer patients with active TB infection.

Keywords: Immunotherapy; lung cancer; programmed cell death 1 (PD-1); programmed cell death-ligand 1 (PD-

L1); tuberculosis (TB)

Submitted Jul 15, 2021. Accepted for publication Sep 16, 2021.

doi: $10.21037 /$ tlcr-21-524

View this article at: https://dx.doi.org/10.21037/tlcr-21-524

\section{Introduction}

Immune checkpoint inhibitors (ICIs) targeting programmed cell death 1 (PD-1) and its ligand (PD-L1) have shifted the paradigm in various solid malignancies, especially in non-small cell lung cancer (NSCLC) (1-6). It is well known that the binding of co-inhibitory receptor PD-1 with its ligand PD-L1 will attenuate the priming, the proliferation and the cytotoxic capacity of $\mathrm{T}$ cells $(7,8)$. Therefore, tumor cells evade host immune surveillance and escape tumor neutralization through the PD-1/PDL1 axis (9). In recent years, immunotherapy with antiPD-1/PD-L1 monoclonal antibodies (mAbs) has made a breakthrough in NSCLC, and the 5-year survival rate of advanced NSCLC has significantly increased from less than $5 \%$ to $23.2 \%(10,11)$.

Immune checkpoint molecules on $\mathrm{T}$ cells, such as $\mathrm{PD}-1$ and cytotoxic T lymphocyte antigen 4 (CTLA-4) will also be up-regulated during chronic infectious diseases. The success of immunotherapy in cancer, especially anti-PD-1/PD$\mathrm{L} 1 \mathrm{mAbs}$ which can restore and enhance the function of $\mathrm{T}$ cells, has aroused people's interest in trying immunotherapy to treat chronic infectious diseases (12). However, growing evidences show anti-PD-1/PD-L1 immunotherapy leads to tuberculosis (TB) reactivation (13-28), which arouse the awareness of physicians about the safety of immunotherapy and immune-related adverse events (irAEs).

According to the 2020 World Health Organization (WHO) global TB report (https://www.who.int/teams/ global-tuberculosis-programme/data), in 2019, an estimated 10.0 million people fell ill with TB, 1.2 million TB deaths among human immunodeficiency virus (HIV)-negative people, and an additional 208,000 deaths among HIVpositive people. China is the second highest TB burden country, accounting for $8.4 \%$ cases in total globally. Accumulating data support the view that TB is a risk factor for the development of lung cancer (29-32), and in
China where both $\mathrm{TB}$ and lung cancer are prevalent, the co-existence of TB with lung cancer is not rare, which brings great challenges to the treatment of patients, especially in the era of tumor immunotherapy. In clinical practice, the following issues are worth noting. Will the excessive activation of $\mathrm{T}$ cells and the release of cytokines induced by anti-PD-1/PD-L1 mAbs activate the latent TB lesions leading to TB recurrence, or deterioration the current TB situation? Will anti-TB therapy increase the incidence of irAEs? There have been many clinical trials on immunotherapy for lung cancer, nonetheless, patients with TB are usually excluded from existing trials resulting in a lack of experience in the above-mentioned issues.

Thus, we retrospectively analyzed 562 patients with advanced lung cancer who received anti-PD-1/ PD-L1 immunotherapy in our hospital from 2015 to 2019, including 13 patients with TB infection prior to immunotherapy (either latent or active TB infection), and analyzed the safety and efficacy of immunotherapy among these patients.

We present the following article in accordance with the STROBE reporting checklist (available at https://dx.doi. org/10.21037/tlcr-21-524).

\section{Methods}

\section{Study design and patient selection}

We retrospectively evaluated a cohort of 562 advanced or recurrent NSCLC patients who received a minimum of one cycle of anti-PD-1/PD-L1 treatment at Shanghai Pulmonary Hospital Affiliated to Tongji University between June 2015 and December 2019. Patients who were confirmed as either latent or active TB infection before the initiation of immunotherapy were enrolled. A total of 13 patients who matched the inclusion criteria were enrolled. Coincidentally, all of the 13 patients were treated 
with anti-PD-1 mAbs. Data on baseline clinicopathological characteristics \{including age, sex, smoking history, cancer type, TNM stage [according to the eighth edition of the TNM staging system (33)], Eastern Cooperative Oncology Group performance status (ECOG PS), driver oncogene alteration, and PD-L1 expression\}, and diagnosis and treatments of TB and cancer (including immunotherapy and other previous therapy) were extracted from the electronic medical records. We recorded the age at the initiation of immunotherapy. Never smokers were defined as persons who had smoked less than 100 cigarettes during their lifetime. This study was approved by the Ethics Committee and Institutional Review Board of Shanghai Pulmonary Hospital.

Additionally, we did a PubMed literature search with the search terms '(tuberculosis OR TB) AND (lung cancer) AND (immunotherapy OR PD-1 OR nivolumab OR pembrolizumab)'. Of the results obtained, each was reviewed for relevance to the topic of TB in the setting of ICIs therapy, as well as relative completeness of the data provided in the cases. Moreover, only articles published in English and human cases were selected.

The study was conducted in accordance with the Declaration of Helsinki (as revised in 2013). The study was approved by ethics board of Shanghai Pulmonary Hospital (No. K18-089-1) and individual consent for this retrospective analysis was waived.

\section{Treatment}

Nivolumab, pembrolizumab, camrelizumab, toripalimab, tislelizumab, and sintilimab were available anti-PD-1 mAbs in mainland China during the study period. All of them were treated with ICIs as monotherapy or in combination with anti-angiogenesis or chemotherapy regardless of treatment lines. Tumor response recorded as partial response (PR), stable disease (SD), or progression disease (PD) was evaluated according to Response Evaluation Criteria in Solid Tumors Version 1.1 (RECIST v1.1) (34). The objective response rate (ORR) was reported as the proportion of patients with complete response or PR, and the disease control rate (DCR) was calculated as the proportion of patients with an objective response or SD (for at least 6 weeks). The diagnosis and grade of irAEs [according to the fourth Common Toxicity Criteria for Adverse Events (CTCAE) classification] were also recorded.

\section{Statistical analysis}

Response to treatment was evaluated using descriptive statistics. Progression-free survival (PFS) was calculated as the time from the first day of immunotherapy until progression of disease or date of death (from any cause), and evaluated using the Kaplan-Meier method. Patients who were still responding to immunotherapy at the censor date (March 1, 2021) or who were lost to follow-up were censored at the last date of follow-up. Statistical analyses were conducted using SPSS software (Version 17.0; SPSS Inc., Chicago, IL, USA).

\section{Results}

\section{Patient characteristics}

We retrospectively reviewed a cohort of 562 advanced or recurrent NSCLC patients who received a minimum of one cycle of anti-PD-1/PD-L1 treatment at Shanghai Pulmonary Hospital Affiliated to Tongji University between June 2015 and December 2019, including 13 patients with the complication of TB infection. Of the 13 patients enrolled (Table 1), 11 (84.6\%) were male and $2(15.4 \%)$ were female with a median age of 62 (range, 42-79) at the initiation of immunotherapy. Seven $(53.8 \%)$ were never smokers, and 6 (46.2\%) were smokers. Eight (61.5\%) were pathologically confirmed as lung squamous cell carcinoma (LUSC), and 5 (38.5\%) were lung adenocarcinoma (LUAD). Of the 9 patients whose driver oncogene alterations were specified, one harbored EGFR 19del, one harbored EGFR $20 \mathrm{ins}$, and the other 7 patients were wild type (WT). Specified PD-L1 expression status was available in 10 of the 13 patients, and 6 of whom were evaluated as PD-L1 positive (one strong positive with PD-L1 $80 \%$ in particular). As for the TNM stage, stage III (IIIb \& IIIc), stage IV, and recurrent NSCLC occurred in $3(23.0 \%), 5$ (38.5\%), and $5(38.5 \%)$ patients, respectively. Only $1(7.7 \%)$ patient was scored ECOG PS 2, the others were all scored 1.

\section{Diagnosis and treatment of $T B$}

Of the 13 patients with TB infection, pulmonary TB occurred in 12 patients (including one tuberculous pleuritis), and extra-pulmonary TB occurred in only one patient (TB of the left hip joint). No patients in our cohort were infected by HIV. Before the initiation of anti-PD-1 treatment, 2 patients were assessed as active pulmonary 


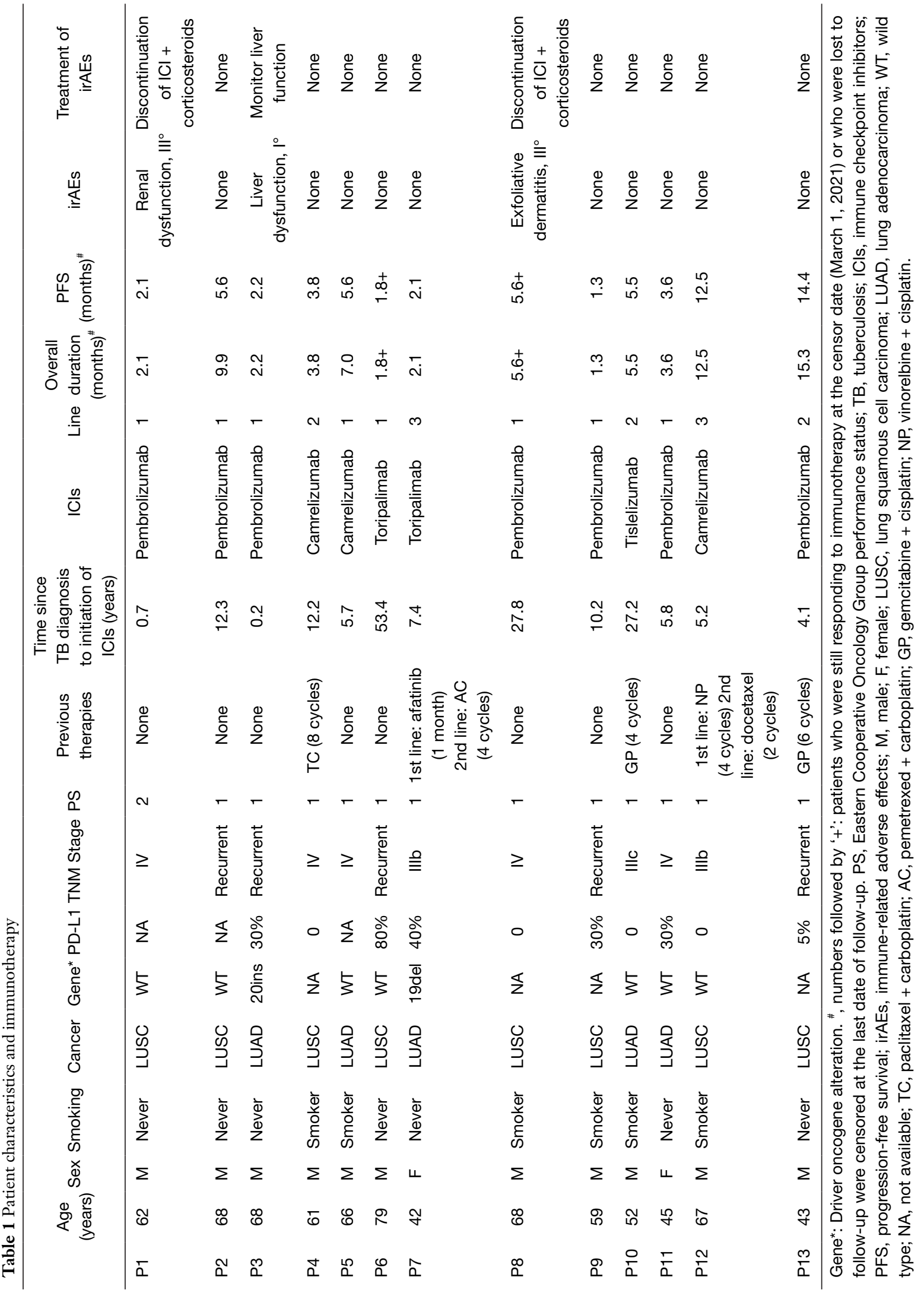


Table 2 Diagnosis and treatment of TB

\begin{tabular}{|c|c|c|c|c|c|c|c|c|c|}
\hline & $\begin{array}{l}\text { TB lesions (organ, } \\
\text { locations) }\end{array}$ & TB-Ab & T-SPOT & $\begin{array}{c}\text { TB } \\
\text { confirmation }\end{array}$ & Calcification & TB cavity & $\begin{array}{c}\text { Active/ } \\
\text { latent TB }\end{array}$ & TB treatment & $\begin{array}{l}\text { Follow-up* } \\
\text { (months) }\end{array}$ \\
\hline $\mathrm{P} 1$ & $\begin{array}{l}\text { Lung, bilateral diffuse } \\
\text { lesions }\end{array}$ & $(+)$ & $(+)$ & Sputum AFB (+) & No & Yes & Active & 4HREZ/4PaReEZLfx & 4.2 \\
\hline $\mathrm{P} 2$ & Lung, left upper & NS & NS & $\begin{array}{l}\text { History + } \\
\text { radiology }\end{array}$ & Yes & No & Latent & NS & 17.3 \\
\hline $\mathrm{P} 4$ & $\begin{array}{l}\text { Lung, bilateral upper and } \\
\text { right lower }\end{array}$ & NS & NS & $\begin{array}{l}\text { History + } \\
\text { radiology }\end{array}$ & No & No & Latent & 2HREZ/4HR & 8.1 \\
\hline P5 & Lung, bilateral upper & NS & NS & $\begin{array}{l}\text { History + } \\
\text { radiology }\end{array}$ & No & No & Latent & 2HREZ/4HR & 12.0 \\
\hline P8 & Lung, bilateral upper & $(-)$ & $(+)$ & $\begin{array}{l}\text { History + } \\
\text { radiology }\end{array}$ & Yes & Yes & Latent & naïve & 5.7 \\
\hline P9 & Lung, right upper & $(-)$ & NS & $\begin{array}{l}\text { History + } \\
\text { radiology }\end{array}$ & No & No & Latent & NS & 9.3 \\
\hline $\mathrm{P} 10$ & Pleura, left lower & NS & NS & $\begin{array}{l}\text { History + } \\
\text { radiology }\end{array}$ & Yes & No & Latent & 3HREZ/9HR & 21.5 \\
\hline $\mathrm{P} 11$ & Lung, right upper & NS & NS & $\begin{array}{l}\text { History + } \\
\text { radiology }\end{array}$ & Yes & No & Latent & 2HREZ/4HR & 3.7 \\
\hline
\end{tabular}

Follow-up*, the time from ICl initiation to the last chest CT with confirmation that there was no deterioration of active TB or reactivation of latent TB at that point of time. TB, tuberculosis; Ab, antibody; NS, not specified; AFB, acid-fast bacillus; H, isoniazid; R, rifampicin; E, ethambutol; Z, pyrazinamide; Pa, pasiniazid; Re, rifapentine; Lfx, levofloxacin.

$\mathrm{TB}$, and the other 11 patients were confirmed as latent TB. The diagnosis of latent TB was mainly based on medical records and imaging findings, all of which had a confirmed TB history, and 10 cases were supported by chest computed tomography (CT) scans. The T-SPOT.TB test was the most commonly used interferon-gamma release assay (IGRA) test at our institution. Among the 5 patients who did T-SPOT. TB test before immunotherapy, 3 patients were confirmed as positive results. Additionally, 2 of the 7 patients who did $\mathrm{TB}$ antibody detection were positive. Of the 12 patients whose chest CT scans were available for pulmonary $\mathrm{TB}$ evaluation, only 2 of whom showed pulmonary TB cavity, and 6 showed calcification lesions. In our cohort, two patients were anti-TB treatment-naïve, and three of them couldn't recall the detailed anti-TB treatment regimens, and the remaining 8 patients all have received 4-drug antiTB treatment. More details of the diagnosis and treatment of TB in our 13 patients are presented in Table 2.

\section{Immunotherapy and other treatments used previously}

All patients enrolled received anti-PD-1 mAbs treatment. Most of them used pembrolizumab ( $n=7), 3$ patients used camrelizumab, 2 toripalimab, and one tislelizumab. Of them, 8 patients received immunotherapy as their firstline treatment, and there was a wide spectrum in types of 

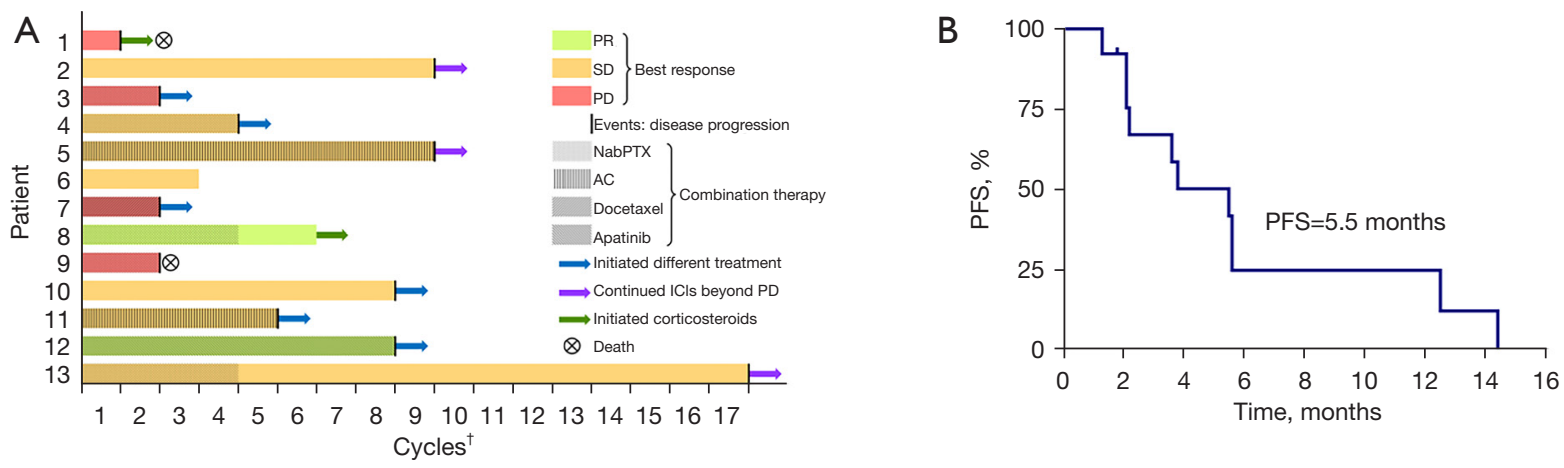

Figure 1 Treatment timelines (A) and progression-free survival of immunotherapy (B) in our cohort. ${ }^{\dagger}$, numbers of cycles of ICIs used till discontinuation for any causes. PR, partial response; SD, stable disease; PD, progression disease; nabPTX, nanoparticle albumin-bound paclitaxel; AC, pemetrexed + carboplatin; ICIs, immune checkpoint inhibitors; PFS, progression-free survival.

other treatments used prior to immunotherapy among the remaining 5 patients (Table 1). All of the 5 patients had received at least 4 cycles of platinum-based double drug chemotherapy, and P7 had received afatinib, a kind of EGFR-tyrosine kinase inhibitor (TKI), as her first-line therapy regimen due to EGFR $19 \mathrm{del}$ alteration. As regards the immunotherapy patterns, more patients received antiPD-1 combination therapies $(n=9)$ than monotherapy $(\mathrm{n}=4)$. In the combination group, 5 patients received antiPD-1 mAbs combined with nanoparticle albumin-bound paclitaxel ( 2 of them turned to monotherapy after 4 cycles of combination therapy due to intolerance), 2 pemetrexed + carboplatin, 1 docetaxel, and 1 apatinib.

\section{The safety and efficacy of immunotherapy}

The median number of anti-PD-1 immunotherapy cycles used in our cohort was 5 (range, 1-17). Neither reactivation of latent $\mathrm{TB}$ nor progression of active $\mathrm{TB}$ was monitored in our cohort during anti-PD-1 immunotherapy. According to the medical records (Table 1), all but two patients (P1 stopped pembrolizumab due to Grade 3 renal dysfunction; P8 stopped pembrolizumab due to Grade 3 exfoliative dermatitis) discontinued anti-PD-1 immunotherapy due to tumor progression. Three patients developed irAEs during anti-PD-1 immunotherapy: Grade 3 renal dysfunction in P1, Grade 1 liver dysfunction in P3, and Grade 3 exfoliative dermatitis in P8. Anti-PD-1 mAbs were withheld and corticosteroids were used in P1 and P8 who were assessed as Grade 3 irAEs. Although immunotherapy was not discontinued in P3, we cautiously monitored patient's liver function results. Diverse tumor responses to anti-PD-1 immunotherapy were evaluated in our cohort (Figure 1A). Of them, the best response was PR in 2 patients, SD in 7 patients, and PD in 4 patients per RECIST v1.1. Thus, the calculated ORR and DCR were $15.4 \%$ and $69.2 \%$, respectively. The median PFS of anti-PD-1 immunotherapy was 5.5 months (Figure 1B). Although the median overall duration of anti-PD-1 immunotherapy was the same as median PFS, three patients (P2, P5, and P13) continued anti-PD-1 immunotherapy for a period beyond PD (PD occurred in extra-pulmonary lesions and pulmonary lesions were stable or still responding).

\section{Concurrent treatments of anti-PD-1 and anti-TB in lung cancer patients complicated with active TB infection}

P1 was a 62-year-old man whose chest CT (November 2017) showed multiple patchy shadows in both lungs, tracheobronchial stenosis in left upper lobe, and soft tissue shadows. Triplicate sputum smear tests were positive $(++)$ for acid-fast bacillus (AFB), and standard anti-TB treatment was initiated using isoniazid, rifampicin, ethambutol, and pyrazinamide (HREZ) from November 20, 2018 without obvious adverse effects. The sputum smear test was negative for AFB in February 2018. Mycobacterium tuberculosis complex was detected by sputum culture in March 2018 and was resistant to streptomycin, isoniazid, and rifampicin. Thus, the anti-TB treatment was changed to pasiniazid, rifapentine, ethambutol, pyrazinamide, and levofloxacin (PaReEZLfx). One month later, another chest CT showed that TB lesions were absorbed remarkably, but the consolidation of the left upper lobe progressed. Later, LUSC was confirmed per the pathology of sputum 

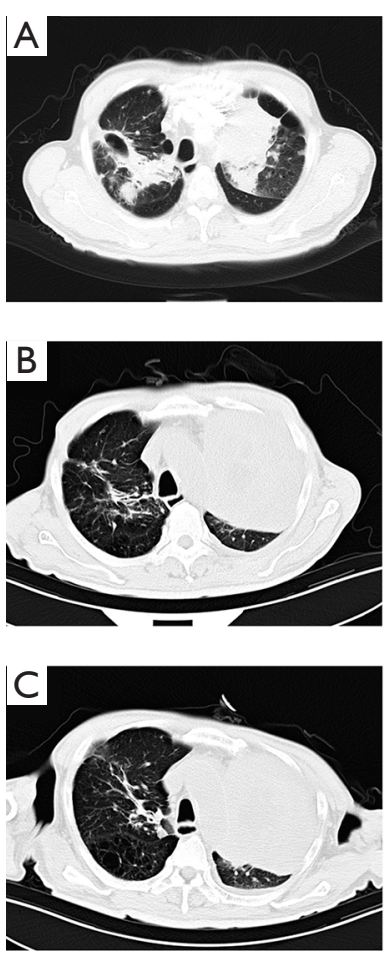

D

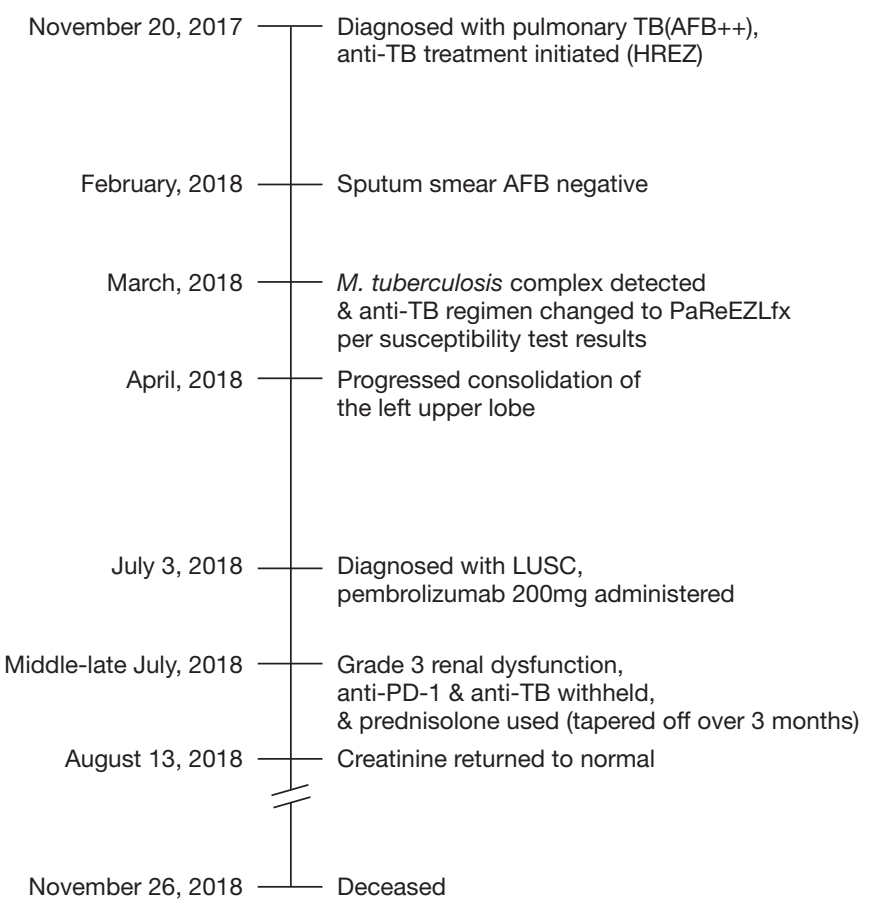

Figure 2 Representative chest CT images at different stages of disease course (A-C) and timeline of therapy and disease status for both lung cancer and TB infection in patient P1 (D). (A) shows patchy shadows in both lungs (including cavity in the right lung), tracheobronchial stenosis in the left upper lobe, and soft tissue shadows. Chest CT images before the initiation of anti-PD-1 immunotherapy (B, TB lesions on the right were absorbed remarkably, but the consolidation of the left upper lobe progressed) and 2 months after the initiation of immunotherapy (C). TB, tuberculosis; AFB, acid-fast bacillus; HREZ, isoniazid, rifampicin, ethambutol, and pyrazinamide; PaReEZLfx, pasiniazid, rifapentine, ethambutol, pyrazinamide, and levofloxacin; LUSC, lung squamous cell carcinoma; PD-1, programmed cell death 1.

samples, and pembrolizumab $200 \mathrm{mg}$ was administered on July 3, 2018. Seven days later, creatinine was $151 \mu \mathrm{mol} / \mathrm{L}$, and then gradually increased to $467 \mu \mathrm{mol} / \mathrm{L}$ (Figure S1). Considering it might be related to immunity disorder, anti-PD-1 immunotherapy and anti-TB treatment were both withheld, and corticosteroid was used (prednisolone, $0.5 \mathrm{mg} / \mathrm{kg} /$ day, and gradually tapered off over a period of approximately 3 months). Creatinine returned to normal on August 13 (Figure S1), and sputum smear tests were negative for AFB repeatedly. Unfortunately, the patient passed away on November 26, 2018 due to complications such as heart failure, severe infectious disease, anemia, and hypoproteinemia. Representative chest CT images at different time points and timeline of therapy and disease status for both lung cancer and TB infection in patient $\mathrm{P} 1$ are summarized in Figure 2.

P3 was a 68-year-old man with the chief complaint of imaging findings of bilateral pulmonary nodules in a health examination (Figure $3 A$ ). After admission, stage
IIIb lung adenocarcinoma with EGFR 20ins alteration was confirmed. Some lesions were dramatically regressed after 4 cycles of chemotherapy, but nodules in the right lung were not changed remarkedly (Figure $3 B$ ). Three nodules in the right lung were resected in October 2018 (Figure 3C). Two of which were lung adenocarcinoma, and the other one in the right lower lobe was AFB (+) granulomas with positive RV0577, IS6110, and $16 \mathrm{sr}$ $R N A$. Isoniazid, rifampicin, ethambutol, and levofloxacin (HRELfx) were administered postoperatively for 2 months and then switched to HR double-drug regimen for 4 months. Approximately 3 months after surgery, the patient developed right supraclavicular lymph node enlargement (Figure 3D) and novel infiltrate in the residual lung, which were metastatic lesions. Pembrolizumab combined with nanoparticle albumin-bound paclitaxel + carboplatin began on January 18, 2019, and tumor progressed after 2 cycles of combination therapy (Figure $3 E$ ). Transaminase was elevated (Grade 1) transiently during immunotherapy. The 

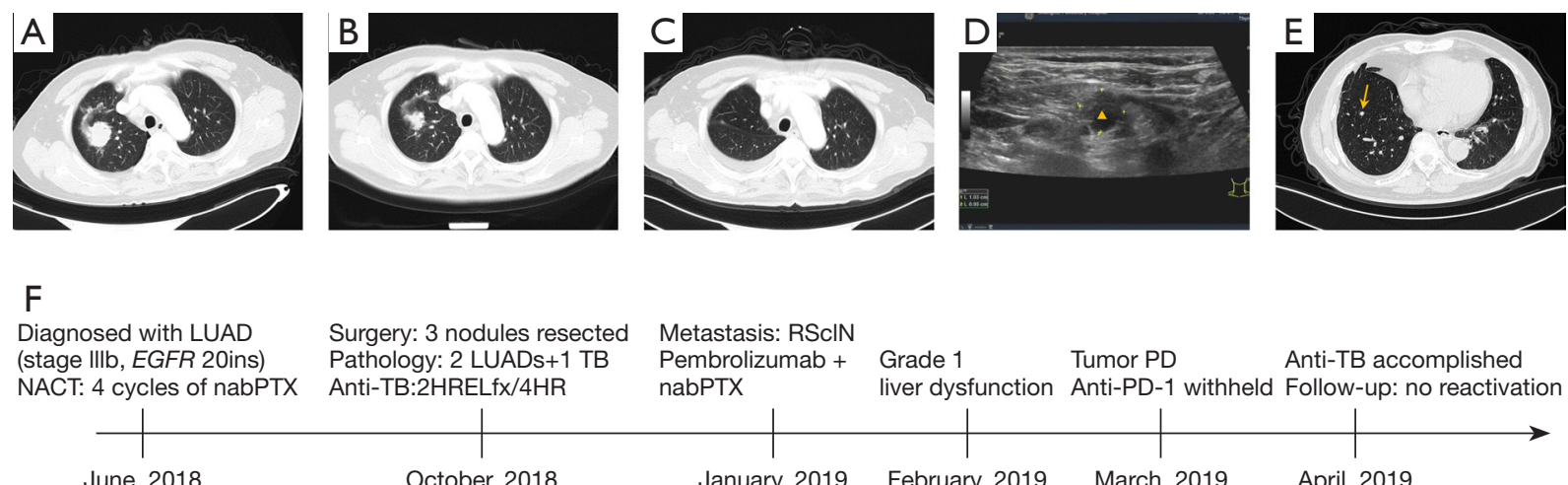

Grade $1 \quad$ Tumor PD

Anti-TB accomplished liver dysfunction Anti-PD-1 withheld Follow-up: no reactivation June, 2018

October, 2018

January, 2019

February, 2019

March, 2019

April, 2019

Figure 3 Representative chest CT or ultrasound images at different stages of disease course (A-E) and timeline of therapy and disease status for both lung cancer and TB infection in patient P3 (F). Chest CT images at the initial diagnosis of lung cancer (A), after 4 cycles of neoadjuvant chemotherapy (B), and after surgery (C). (D) shows the ultrasound image of the enlarged right supraclavicular lymph node (marked with a triangle) that was confirmed as a metastatic lesion afterwards. (E) shows a novel infiltrate (marked with an arrow) after 2 cycles of immunotherapy. LUAD, lung adenocarcinoma; NACT, neoadjuvant chemotherapy; nabPTX, nanoparticle albumin-bound paclitaxel; TB, tuberculosis; HRELfx, isoniazid, rifampicin, ethambutol, and levofloxacin; HR, isoniazid and rifampicin; RSclN, right supraclavicular lymph node; PD, progression disease; PD-1, programmed cell death 1.

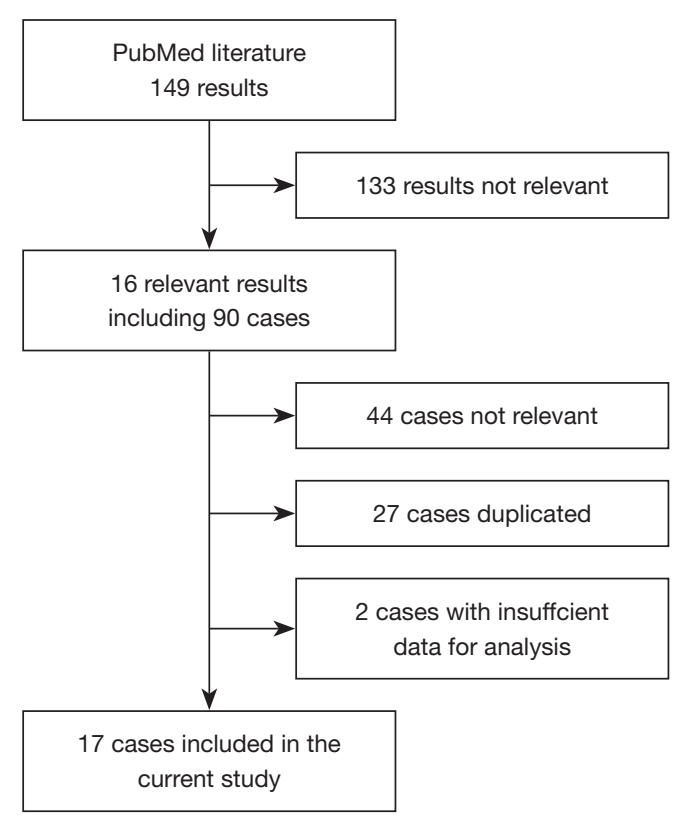

Figure 4 A flowchart of case inclusion.

timeline of therapy and disease status for both lung cancer and TB infection in patient $\mathrm{P} 3$ is represented in Figure $3 F$.

\section{Summary of literature reviews}

PubMed literature reviews on the topic were done per the protocol mentioned above. In total, 17 eligible cases were included, and a flowchart of case inclusion is presented (Figure 4). In brief, most patients enrolled were male (14/17, $82.4 \%)$, Asian (11/16, 68.8\%), LUAD (11/17, 64.7\%), and stage IV $(10 / 13,76.9 \%)$. The number of patients no less than 65 years old was similar to those under 65. AntiPD-1 mAbs used in those cases were limited to nivolumab $(10 / 17,58.8 \%)$ and pembrolizumab (7/17, 41.2\%), and they were all administered alone except for one case of combined chemotherapy. More patients $(n=10)$ received immunotherapy as non-first-line treatments, and the median cycles of immunotherapy used till TB diagnosis in all cases were 5.5 (range, 2-41). Although the overwhelming cases were pulmonary TB $(13 / 17,76.5 \%)$, extra-pulmonary TB infections were also found in the sites of pleura, pericardium, and bone. All of the cases were given anti-TB treatment (details are provided in Table 3 if available), and immunotherapy was not suspended only in one case, and corticosteroids were used in 6 out of the 8 patients with relevant records. Eight cases reported the outcomes of TB, and one died shortly after a second operation for spinal cord compression, and the rest were controlled or improved clinically by medication.

\section{Discussion}

With the approval of immunotherapy for treating malignancies, it does benefit certain patients with the median PFS of cancer immunotherapy reaching over 10 months 


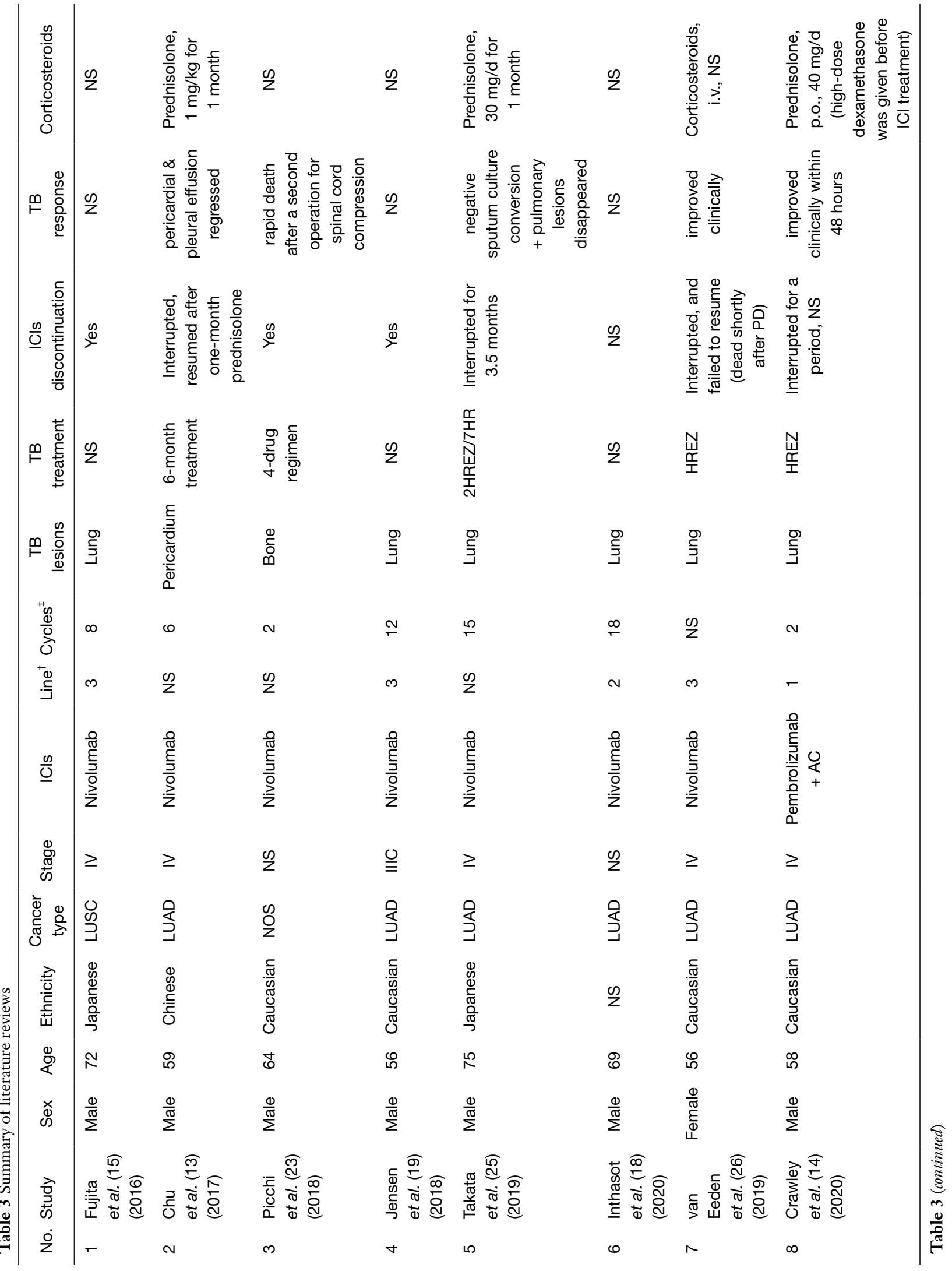




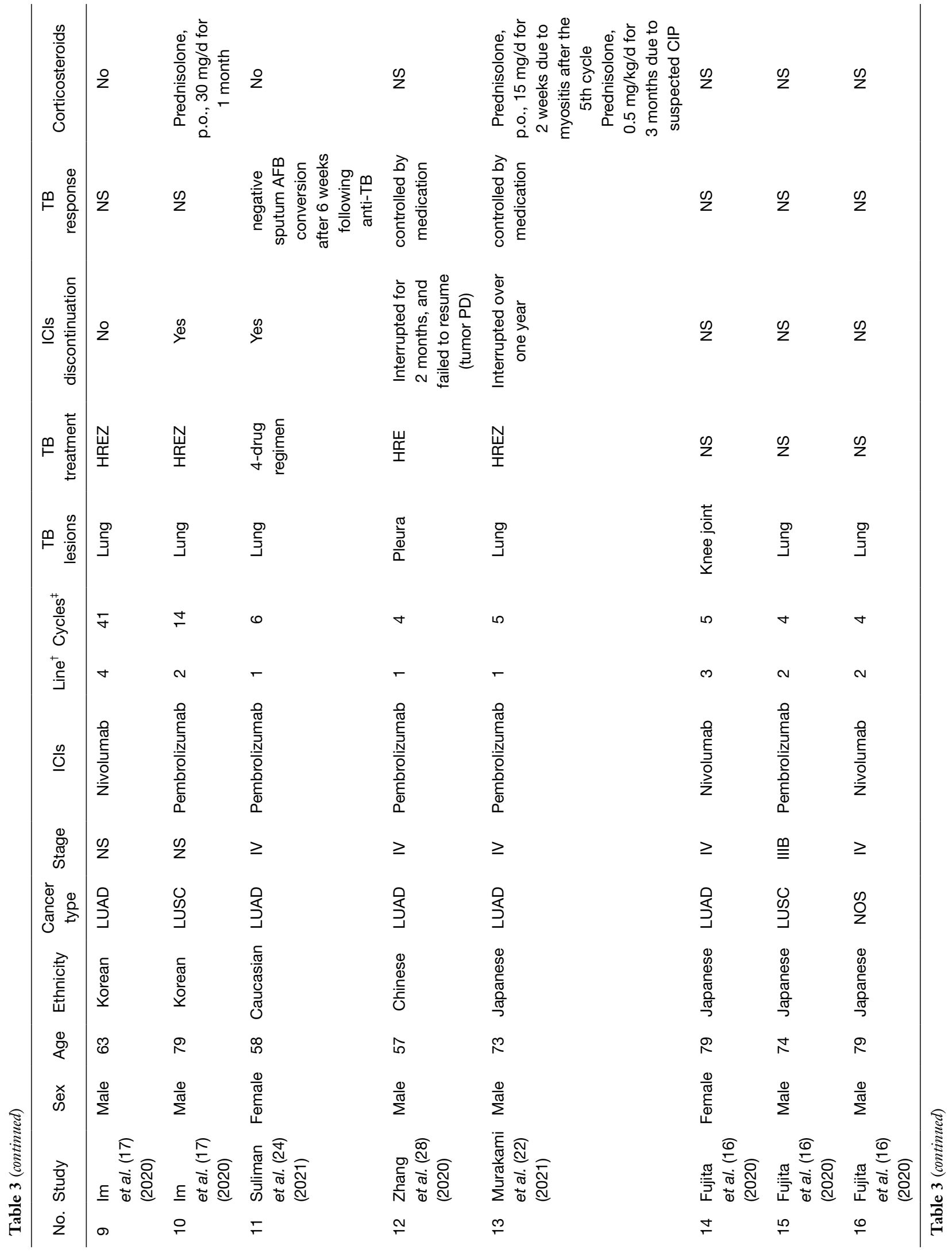




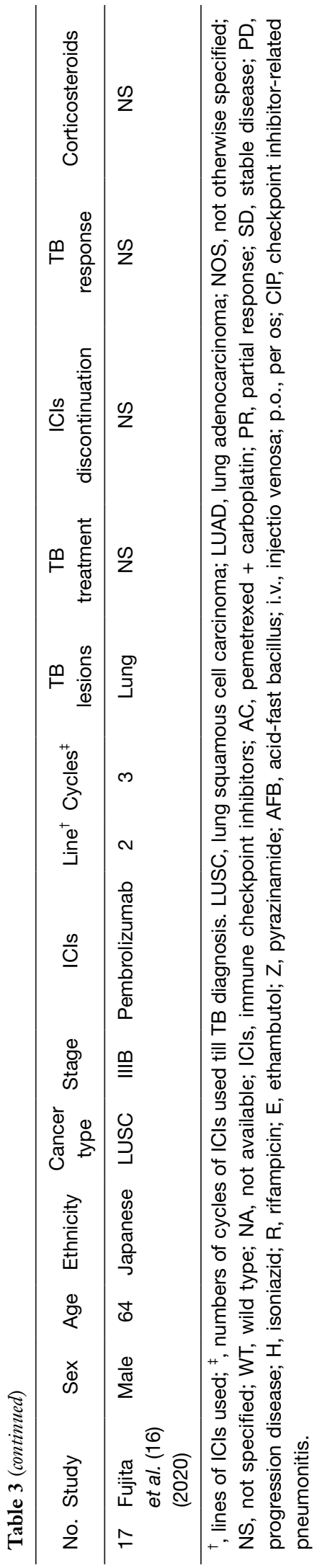

(4,35-39), even over 20 months (40) in advanced NSCLC. However, immunotherapy with ICIs can lead to a variety of immunotoxicities by unbalancing the immune system. As we know, TB is an infectious disease that is closely related to the immune system and regarded as a risk factor for lung cancer which is supported by the carcinogenous roles of chronic inflammation and pulmonary fibrosis $(41,42)$. Accumulating cases of TB diagnosis after administering anti-PD-1/PDL1 mAbs are being reported recently. However, the safety and efficacy of anti-PD-1/PD-L1 treatment in lung cancer patients complicated with TB infection is seldom reported.

Regarding the safety of anti-PD-1 immunotherapy in our study, both patients with active TB experienced irAEs, and one died shortly though discontinuation of pembrolizumab and administration of prednisolone were applied. Meanwhile, only one patient with latent TB experienced irAE during immunotherapy and got improved clinically by appropriate treatment strategies. Fortunately, no reactivation of latent $\mathrm{TB}$ or deterioration of active TB occurred during immunotherapy. As regards the efficacy, the median PFS was 5.5 months, and the PFS was shorter numerically in the two patients with active TB infection (2.1 and 2.2 months) than that in the others. However, given the heterogeneous treatment patterns and lines within a small sample cohort, the reported data on survival is not of much reference value.

Another 17 cases reported online were also reviewed (online cohort), but it is not clear whether the patients were infected with TB before immunotherapy. All cases in online cohort were diagnosed with TB during immunotherapy, and we could deduce that most patients were confirmed TB infection within 6 months according to the cycles of ICIs used till TB diagnosis, which suggests preexisting latent $\mathrm{TB}$ in those patients. Regarding the treatment, immunotherapy was suspended with corticosteroids being administered simultaneously in most patients, and all cases were improved except one who died shortly after surgery. However, it is not made clear whether the death was attributed to the deterioration of TB.

To our knowledge, $\mathrm{T}$ cells play an important role in both cancer and pathogen immunity. PD-1/PD-L1 blockade could unleash the cytotoxic capacity of T cells, and some people would take it for granted that immunotherapy with anti-PD-1/PD-L1 mAbs brought the theoretical benefit in treating infectious diseases. However, growing evidences of $\mathrm{TB}$ reactivation after anti-PD-1/PD-L1 treatment break the hypothesis.

In a preclinical study performed by Lázár-Molnár 
and colleagues (43), a conclusion is drawn that the PD-1 pathway is essential to control the excessive inflammatory responses after TB infection. They found that after TB infection, focal necrosis and dominant neutrophils infiltration were the distinguishing pathological features in the lungs of PD-1 deficiency mice compared with control. Moreover, certain proinflammatory cytokines were significantly increased in PD-1 deficiency mice, which further affirmed the viewpoint of excessive inflammatory responses. Interestingly, PD-1 deficiency mice have significantly poorer survival after TB infection than control.

Clinically, Fujita et al. (15) collected transbronchial lung biopsy specimens from a patient who developed TB after anti-PD-1 immunotherapy, and histopathological findings showed diffuse lymphocyte infiltrations in the alveolar area suggesting excessive inflammatory responses. Collectively, excessive inflammatory responses and necrosis are the main characteristics pathologically both in mice and human, which is similar to immune reconstitution inflammatory syndrome in patients treated with anti-HIV therapies (44).

Furthermore, Tezera et al. (45) explored the mechanisms of TB reactivation after anti-PD-1 immunotherapy. First, they found that PD-1 was expressed in lung tissues infected by TB but not the areas of immunotherapy, and was upregulated after TB infection. Once PD-1 was blocked by anti-PD-1 mAbs, both TB growth and cytokine secretion [especially tumor necrosis factor alpha (TNF- $\alpha$ )] increased. And such phenomena would be reversed by TNF- $\alpha$ neutralization. Consequently, they reached the conclusions that the immune response in TB was regulated by PD1, and PD-1 blockade facilitated TB growth via excessive TNF- $\alpha$ secretion.

There are several limitations in our study. The first is the nature of retrospective research. Next, the sample size is not enough in our cohort to make convincing conclusions that can affect decision-making in clinical practice. Although patients with active TB infection prior to immunotherapy suffered poor survivals in our cohort, the feasibility and appropriate timing of immunotherapy in such patients are still to be defined. Besides, whether corticosteroids contribute to immunosuppression leading to the blunting effects of immunotherapy needs further discussion. Most importantly, more case reports and exploratory experiments are warranted to uncover the mechanisms which are conducive to precise prevention and treatment in the future.

\section{Conclusions}

According to our study, immunotherapy is relatively safe for lung cancer patients complicated with previously treated latent TB infection, and the efficacy of immunotherapy in this specified population is not inferior to that in lung cancer patients without TB infection. However, it is being widely accepted that the blockade of PD-1/PD-L1 is associated with the development or reactivation of TB. Thus, the screening for TB using IGRA prior to anti-PD-1/ PD-L1 immunotherapy is of great importance, especially in TB epidemic regions and immunosuppressive patients. Besides, irAEs should be monitored more cautiously in lung cancer patients complicated with active TB infection due to possible poor compliance with and attenuated efficacy of anti-PD-1/PD-L1 immunotherapy led by irAEs.

\section{Acknowledgments}

Funding: This work was supported by grants from National Natural Science Foundation of China (No. 81902314); Science and Technology Commission of Shanghai Municipality (No. 19411950300); Clinical Research Plan of Shanghai Hospital Development Center (No. SHDC2020CR4001); Natural Science Foundation of Shanghai (No. 20ZR1447100); and Scientific Research Project of Shanghai Pulmonary Hospital (No. fkcx1903).

\section{Footnote}

Reporting Checklist: The authors have completed the STROBE reporting checklist. Available at https://dx.doi. org/10.21037/tlcr-21-524

Data Sharing Statement: Available at https://dx.doi. org/10.21037/tlcr-21-524

Conflicts of Interest: All authors have completed the ICMJE uniform disclosure form (available at https://dx.doi. org/10.21037/tlcr-21-524). CZ and YH serve as unpaid editorial board members of Translational Lung Cancer Research. The other authors have no conflicts of interest to declare.

Ethical Statement: The authors are accountable for all aspects of the work in ensuring that questions related to the accuracy or integrity of any part of the work are 
appropriately investigated and resolved. The study was conducted in accordance with the Declaration of Helsinki (as revised in 2013). The study was approved by ethics board of Shanghai Pulmonary Hospital (No. K18-089-1) and individual consent for this retrospective analysis was waived.

Open Access Statement: This is an Open Access article distributed in accordance with the Creative Commons Attribution-NonCommercial-NoDerivs 4.0 International License (CC BY-NC-ND 4.0), which permits the noncommercial replication and distribution of the article with the strict proviso that no changes or edits are made and the original work is properly cited (including links to both the formal publication through the relevant DOI and the license). See: https://creativecommons.org/licenses/by-nc-nd/4.0/.

\section{References}

1. Brahmer J, Reckamp KL, Baas P, et al. Nivolumab versus Docetaxel in Advanced Squamous-Cell Non-Small-Cell Lung Cancer. N Engl J Med 2015;373:123-35.

2. Herbst RS, Baas P, Kim DW, et al. Pembrolizumab versus docetaxel for previously treated, PD-L1positive, advanced non-small-cell lung cancer (KEYNOTE-010): a randomised controlled trial. Lancet 2016;387:1540-50.

3. Paz-Ares L, Luft A, Vicente D, et al. Pembrolizumab plus Chemotherapy for Squamous Non-Small-Cell Lung Cancer. N Engl J Med 2018;379:2040-51.

4. Reck M, Rodríguez-Abreu D, Robinson AG, et al. Pembrolizumab versus Chemotherapy for PD-L1Positive Non-Small-Cell Lung Cancer. N Engl J Med 2016;375:1823-33.

5. Rittmeyer A, Barlesi F, Waterkamp D, et al. Atezolizumab versus docetaxel in patients with previously treated non-small-cell lung cancer (OAK): a phase 3, openlabel, multicentre randomised controlled trial. Lancet 2017;389:255-65.

6. Zhou C, Chen G, Huang Y, et al. Camrelizumab plus carboplatin and pemetrexed versus chemotherapy alone in chemotherapy-naive patients with advanced non-squamous non-small-cell lung cancer (CameL): a randomised, openlabel, multicentre, phase 3 trial. Lancet Respir Med 2021;9:305-14.

7. Chen DS, Mellman I. Oncology meets immunology: the cancer-immunity cycle. Immunity 2013;39:1-10.

8. Pardoll DM. The blockade of immune checkpoints in cancer immunotherapy. Nat Rev Cancer 2012;12:252-64.
9. Mellman I, Coukos G, Dranoff G. Cancer immunotherapy comes of age. Nature 2011;480:480-9.

10. Borghaei H, Gettinger S, Vokes EE, et al. Five-Year Outcomes From the Randomized, Phase III Trials CheckMate 017 and 057: Nivolumab Versus Docetaxel in Previously Treated Non-Small-Cell Lung Cancer. J Clin Oncol 2021;39:723-33.

11. Garon EB, Hellmann MD, Rizvi NA, et al. Five-Year Overall Survival for Patients With Advanced Non-SmallCell Lung Cancer Treated With Pembrolizumab: Results From the Phase I KEYNOTE-001 Study. J Clin Oncol 2019;37:2518-27.

12. Wykes MN, Lewin SR. Immune checkpoint blockade in infectious diseases. Nat Rev Immunol 2018;18:91-104.

13. Chu YC, Fang KC, Chen HC, et al. Pericardial Tamponade Caused by a Hypersensitivity Response to Tuberculosis Reactivation after Anti-PD-1 Treatment in a Patient with Advanced Pulmonary Adenocarcinoma. J Thorac Oncol 2017;12:e111-4.

14. Crawley D, Breen RA, Elkington PT, et al. Tuberculosis associated with Triplet therapy for lung cancer. Thorax 2020;75:609-10.

15. Fujita K, Terashima T, Mio T. Anti-PD1 Antibody Treatment and the Development of Acute Pulmonary Tuberculosis. J Thorac Oncol 2016;11:2238-40.

16. Fujita K, Yamamoto $Y$, Kanai $O$, et al. Incidence of Active Tuberculosis in Lung Cancer Patients Receiving Immune Checkpoint Inhibitors. Open Forum Infect Dis 2020;7:ofaa126.

17. Im Y, Lee J, Kim SJ, et al. Development of tuberculosis in cancer patients receiving immune checkpoint inhibitors. Respir Med 2020;161:105853.

18. Inthasot V, Bruyneel M, Muylle I, et al. Severe pulmonary infections complicating nivolumab treatment for lung cancer: a report of two cases. Acta Clin Belg 2020;75:308-10.

19. Jensen KH, Persson G, Bondgaard AL, et al. Development of pulmonary tuberculosis following treatment with anti-PD-1 for non-small cell lung cancer. Acta Oncol 2018;57:1127-8.

20. Kato $Y$, Watanabe $Y$, Yamane $Y$, et al. Reactivation of TB during administration of durvalumab after chemoradiotherapy for non-small-cell lung cancer: a case report. Immunotherapy 2020;12:373-8.

21. Langan EA, Graetz V, Allerheiligen J, et al. Immune checkpoint inhibitors and tuberculosis: an old disease in a new context. Lancet Oncol 2020;21:e55-65.

22. Murakami S, Usui R, Nakahara Y, et al. Readministration of Pembrolizumab after Treatment of Tuberculosis 
Activated by Initial Pembrolizumab Therapy. Intern Med 2021;60:1743-6.

23. Picchi H, Mateus C, Chouaid C, et al. Infectious complications associated with the use of immune checkpoint inhibitors in oncology: reactivation of tuberculosis after anti PD-1 treatment. Clin Microbiol Infect 2018;24:216-8.

24. Suliman AM, Bek SA, Elkhatim MS, et al. Tuberculosis following programmed cell death receptor-1 (PD-1) inhibitor in a patient with non-small cell lung cancer. Case report and literature review. Cancer Immunol Immunother 2021;70:935-44.

25. Takata S, Koh G, Han Y, et al. Paradoxical response in a patient with non-small cell lung cancer who received nivolumab followed by anti-Mycobacterium tuberculosis agents. J Infect Chemother 2019;25:54-8.

26. van Eeden R, Rapoport BL, Smit T, et al. Tuberculosis Infection in a Patient Treated With Nivolumab for Nonsmall Cell Lung Cancer: Case Report and Literature Review. Front Oncol 2019;9:659.

27. Zaemes J, Kim C. Immune checkpoint inhibitor use and tuberculosis: a systematic review of the literature. Eur J Cancer 2020;132:168-75.

28. Zhang YB, Liu SJ, Hu ZD, et al. Increased Th17 activation and gut microbiota diversity are associated with pembrolizumab-triggered tuberculosis. Cancer Immunol Immunother 2020;69:2665-71.

29. Brenner DR, Boffetta P, Duell EJ, et al. Previous lung diseases and lung cancer risk: a pooled analysis from the International Lung Cancer Consortium. Am J Epidemiol 2012;176:573-85.

30. Leung CC, Hui L, Lee RS, et al. Tuberculosis is associated with increased lung cancer mortality. Int J Tuberc Lung Dis 2013;17:687-92.

31. Liang HY, Li XL, Yu XS, et al. Facts and fiction of the relationship between preexisting tuberculosis and lung cancer risk: a systematic review. Int J Cancer 2009;125:2936-44.

32. Yu YH, Liao CC, Hsu WH, et al. Increased lung cancer risk among patients with pulmonary tuberculosis: a population cohort study. J Thorac Oncol 2011;6:32-7.

33. Detterbeck FC, Boffa DJ, Kim AW, et al. The Eighth Edition Lung Cancer Stage Classification. Chest 2017;151:193-203.

34. Eisenhauer EA, Therasse P, Bogaerts J, et al. New response evaluation criteria in solid tumours: revised RECIST guideline (version 1.1). Eur J Cancer 2009;45:228-47.
35. Antonia SJ, Villegas A, Daniel D, et al. Overall Survival with Durvalumab after Chemoradiotherapy in Stage III NSCLC. N Engl J Med 2018;379:2342-50.

36. Antonia SJ, Villegas A, Daniel D, et al. Durvalumab after Chemoradiotherapy in Stage III Non-Small-Cell Lung Cancer. N Engl J Med 2017;377:1919-29.

37. Jotte R, Cappuzzo F, Vynnychenko I, et al. Atezolizumab in Combination With Carboplatin and Nab-Paclitaxel in Advanced Squamous NSCLC (IMpower131): Results From a Randomized Phase III Trial. J Thorac Oncol 2020;15:1351-60.

38. Nishio M, Barlesi F, West H, et al. Atezolizumab Plus Chemotherapy for First-Line Treatment of Nonsquamous NSCLC: Results From the Randomized Phase 3 IMpower132 Trial. J Thorac Oncol 2021;16:653-64.

39. Socinski MA, Jotte RM, Cappuzzo F, et al. Atezolizumab for First-Line Treatment of Metastatic Nonsquamous NSCLC. N Engl J Med 2018;378:2288-301.

40. Langer CJ, Gadgeel SM, Borghaei H, et al. Carboplatin and pemetrexed with or without pembrolizumab for advanced, non-squamous nonsmall-cell lung cancer: a randomised, phase 2 cohort of the open-label KEYNOTE-021 study. Lancet Oncol 2016;17:1497-508.

41. Ardies CM. Inflammation as cause for scar cancers of the lung. Integr Cancer Ther 2003;2:238-46.

42. Coussens LM, Werb Z. Inflammation and cancer. Nature 2002;420:860-7.

43. Lázár-Molnár E, Chen B, Sweeney KA, et al. Programmed death-1 (PD-1)-deficient mice are extraordinarily sensitive to tuberculosis. Proc Natl Acad Sci U S A 2010;107:13402-7.

44. Lawn SD, Bekker LG, Miller RF. Immune reconstitution disease associated with mycobacterial infections in HIVinfected individuals receiving antiretrovirals. Lancet Infect Dis 2005;5:361-73.

45. Tezera LB, Bielecka MK, Ogongo P, et al. Anti-PD-1 immunotherapy leads to tuberculosis reactivation via dysregulation of TNF- $\alpha$. Elife 2020;9:52668.

Cite this article as: Shi J, Li J, Wang Q, Cheng X, Du H, Han R, Li X, Zhao C, Gao G, He Y, Chen X, Su C, Ren S, Wu F, Zhang Z, Zhou C. The safety and efficacy of immunotherapy with antiprogrammed cell death 1 monoclonal antibody for lung cancer complicated with Mycobacterium tuberculosis infection. Transl Lung Cancer Res 2021;10(10):3929-3942. doi: 10.21037/tlcr21-524 


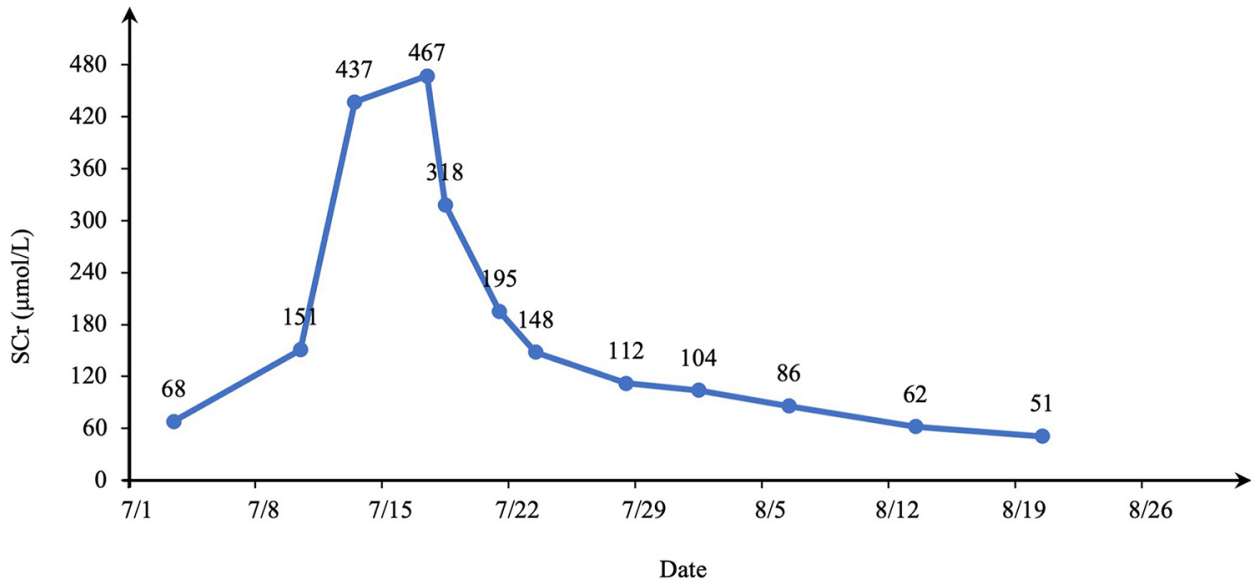

\begin{tabular}{|c|c|c|c|c|c|c|c|c|c|c|c|c|}
\cline { 2 - 13 } \multicolumn{1}{c|}{} & $7 / 3$ & $7 / 10$ & $7 / 13$ & $7 / 17$ & $7 / 18$ & $7 / 21$ & $7 / 23$ & $7 / 28$ & $8 / 1$ & $8 / 6$ & $8 / 13$ & $8 / 20$ \\
\hline $\mathrm{SCr}(\mu \mathrm{mol} / \mathrm{L})$ & 68 & 151 & 437 & 467 & 318 & 195 & 148 & 112 & 104 & 86 & 62 & 51 \\
\hline
\end{tabular}

Figure S1 Line chart of serum creatinine over time. 